\title{
Use of Rational Numbers in the Design of Robust Geometric Primitives for Three-Dimensional Spatial Database Systems
}

\author{
Brian E. Weinrich \& Markus Schneider* \\ University of Florida \\ Department of Computer \& Information Science \& Engineering \\ Gainesville, FL 32611, USA \\ \{brianew, mschneid\}@cise.ufl.edu
}

\begin{abstract}
A necessary step in the implementation of three-dimensional spatial data types for spatial database systems and GIS is the development of robust geometric primitives. The authors have previously shown the need for 3D spatial data types and rigorously defined them. In this paper, we propose a set of $3 \mathrm{D}$ geometric primitives that can be used to implement them robustly. We provide for their robustness by specifying them using rational numbers. In the discretization of space, the developers of two-dimensional spatial data types have used simplicial complexes, realms or dual grids to produce robustness, but extending any of these to $3 \mathrm{D}$ is not adequate. Furthermore, rational number theory is sufficiently developed to apply to 3D implementation primitives. Efforts are lacking, however, in the field of spatial databases to show that spatial operations involving 3D spatial data types are closed under rational arithmetic. We therefore define four geometric primitives using rational numbers: point, segment, facet and solid which correspond to $0 \mathrm{D}$, 1D, 2D and 3D spatial objects respectively. Also, we compare the rational specification of $3 \mathrm{D}$ primitives to the discretization methods used in 2D. Finally, we show that intersections involving these primitives have rational closure. We therefore conclude that use of rational numbers in the design of geometric primitives provides for a robust implementation of three-dimensional spatial data types.
\end{abstract}

Categories and Subject Descriptors: H.2.8 Database Applications - Spatial Databases and GIS.

General Terms: Design, Algorithms, Theory.

Keywords: geometric primitives, rational numbers, discrete model, 3D spatial data types, spatial database, GIS.

\section{INTRODUCTION}

The development of a three-dimensional spatial database system requires determining how to robustly represent three-dimensional spatial data types in computer memory. Spatial database systems

*This work was partially supported by the National Science Foundation under grant number NSF-CAREER-IIS-0347574.

Permission to make digital or hard copies of all or part of this work for personal or classroom use is granted without fee provided that copies are not made or distributed for profit or commercial advantage and that copies bear this notice and the full citation on the first page. To copy otherwise, to republish, to post on servers or to redistribute to lists, requires prior specific permission and/or a fee.

GIS'05, November 4, 2005, Bremen, Germany.

Copyright 2005 ACM 1-59593-146-5/05/0011 ...\$5.00. have more demanding robustness requirements than standard programming languages can provide. Implementations of both the definitions of spatial data types and spatial operations must produce results that are much closer to exactness than is possible in floating or fixed point operations. They must especially provide for topological correctness. We will propose in this paper that implementers of 3D spatial database systems use arbitrary precision rational arithmetic rather than simplicial complexes, realms or dual grids that the developers of two-dimensional database systems have used.

Developing a robust implementation of 3D spatial data types initially requires two things. First of all, we must specify $3 \mathrm{D}$ geometric primitives through which we can represent the data types in computer storage. We will specify them using rational numbers so that we can use an arbitrary precision rational number system to perform high precision arithmetic in the spatial database system.

Secondly, for rational arithmetic to be feasible for 3D spatial database systems, spatial operations in 3D must have rational closure. Thus, we will show that the primitives have rational intersections. Since mathematicians long ago proved the closure of rational arithmetic, we will therefore show that a 3D spatial database system is closed for both arithmetic and spatial operations when using rational numbers.

This work is an important step in the implementation of 3D spatial data types. We proceed in the following way. Section 2 discusses related work. Section 3 presents the 3D geometric implementation primitives. Section 4 shows the rational closure of intersections of the 3D primitives. Section 5 draws conclusions and discusses future work.

\section{RELATED WORK IN DESIGN, DISCRETIZATION AND RATIONAL NUMBERS}

The work presented in this paper has as a basis work that has been done in three areas related to the robust implementation of 3D spatial database systems. These three areas are the design of abstract three-dimensional spatial data types, the discretization of spatial objects and the use of rational numbers. We now proceed one by one to discuss these related areas.

If we are to talk about implementation issues for abstract 3D spatial data types and about robustness in particular, their design must exist so that it is clear what we are implementing. It must also be clear that they are important. The authors' work in [9] provides a comprehensive and rigorous definition of 3D data types. In [9], we rigorously define the data types and specify geometric spatial operations. We also reveal a need for them. We intend this modeling 
of the 3D spatial data types as a first step in developing them. We give both unstructured and structured definitions of the data types which are point $3 D$, line $3 D$, surface, relief and volume. We refer the reader to [9] for the definitions of the abstract data types and other detailed information on our abstract design.

It is impossible to exactly represent the continuous boundaries or interiors of spatial objects in finite computer memory. Like the implementers of 2D spatial data types have done, we must therefore use simple objects called geometric primitives to form discretized approximations of 3D spatial objects. When we specify 3D geometric primitives, we must define operations including predicates which give relationships between the primitives. The work reported in [12] performs the important task of classifying 3D topological relationships between spatial objects using the 9-intersection model, but does not deal with primitives and does not provide precise mathematical formulas for operations between primitives.

To discretize spatial objects, we must also discretize space. The developers of 2D systems have used simplicial complexes or realms to discretize space and have suggested the use of dual grids. We therefore now review some literature on the use of simplicial complexes, realms or dual grids in 2D spatial database systems.

The discussion in [6] provides an excellent overview of simplicial complexes and realms in 2D spatial database systems. Simplicial complexes have shortcomings which [8] points out. Their independence from computer arithmetic makes it difficult to connect them to finite arithmetic and spatial databases. Also, the triangular networks formed by the 2-complexes contain more information than is needed for a spatial database system.

Thus, many current implementations of 2D spatial database systems rely on realms which were originally developed in [8]. A realm in $2 \mathrm{D}$ is a grid with points and line segments. The grid points and line segments are geometric primitives for 2D spatial data types. The work in [8] provides a thorough, robust design and implementation of realms for a $2 \mathrm{D}$ spatial database system. Although use of realms overcomes the problems of simplicial complexes, it has some problems of its own. Most of these problems relate to the redrawing process required when a spatial object is updated or inserted into a database. See [3] for a treatment of these issues. These problems are minor relative to the problems solved by realms, but they invite attempts at improvements.

Dual grids, as proposed in [3], rely on realms also. However, two grids are formed instead of only one, a grid for point objects and a grid for line segments. Dual grids even use rational numbers to obtain exact representations of the grid points. Use of dual grids has the potential to overcome many of the problems of realms.

Developers have successfully used realms and simplicial complexes to implement 2D spatial databases. Dual grids also show promise. It is not adequate, however, to extend these discretization methods to three dimensions. Realms have closure problems when extended to 3D. When using realms, the intersection of a line and plane must occur at a grid point of the realm. However, even the nearest realm grid point in 3D is probably not in the plane. With the rational implementation that we propose in this paper, we essentially have no grid beyond what the limits of finite computer memory impose on an arbitrary precision rational number system. We discuss more later on how the use of rational numbers in 3D provides a better discretization method and, thus, better robustness.

Next, we discuss what has been done to use rational numbers to produce robust results in spatial systems. To do so, we must answer two questions. First of all, what arbitrary precision rational number arithmetic packages have been developed and are they adequate? Also, what have spatial database researchers done to determine how rational software handles the intersection of $3 \mathrm{D}$ spatial objects?
In the past ten years researchers in computational geometry have done a lot of development work with rational software with a view towards applying the software to spatial objects. The evidence presented in [11] shows that research and development in computational geometry was headed in that direction ten years ago. Since then, developers have produced packages such as MAPC [7], PARI [1], apfloat [10] and rational arithmetic components within both LEDA and CGAL [2], to name a few. The cited papers report on the robustness of the rational arithmetic in these systems. Thus, plenty of adequate rational systems exist.

It is, of course, possible to perform robust arithmetic using rational numbers because they are both dense and closed under the basic arithmetic operations. What about spatial operations involving a line or plane in three dimensions? This is an important question because two of the 3D geometric primitives (which we present in Section 3) come from finite restrictions of lines and planes. The spatial database community has seen some work applying rational numbers to the representation of spatial objects. The work in [5, $4,3]$ shows many are aware of the possibilities of rational representation of geometric primitives in both $2 \mathrm{D}$ and 3D. In order for rational specification of $3 \mathrm{D}$ geometrics primitives to produce a robust 3D spatial database system, however, the intersections of the primitives must be rational. We are not aware of any work within the spatial database community showing that the intersections of 3D geometric primitives are rational. Therefore, after defining the 3D primitives in the next section (Section 3), we will prove that they have rational intersections in Section 4.

\section{GEOMETRIC PRIMITIVES FOR THREE DIMENSIONS}

Geometric primitives are simple objects that are used to approximate and implement spatial objects in computer storage. The geometric primitives that we will specify for three-dimensional spatial databases systems are, intuitively, single points, line segments, plane segments and solids in 3D space. By plane segments, we mean a finite, contiguous part of a plane. Single points represent a point $3 D$ object and many small line segments approximate a curved line $3 D$ object. A collection of plane segments approximate a surface, relief or a boundary of a volume. One or more solids approximate a volume. So, we can specify all five abstract 3D spatial data types using the four $3 \mathrm{D}$ primitives that we are about to specify.

We name the 3D geometric primitives point, segment, facet and solid. Note that since these objects are primitives, they do not need to have holes or multi-component parts. It is the abstract spatial data types that must provide for holes and multi-component parts. Rather, we represent a hole in a surface, relief or volume using a primitive. Furthermore, we use a set of primitives to represent each component in an object that has multi-component parts.

In theory, we would like to specify the primitives using the abstract set of rational numbers $\mathbb{Q}$. In practice, this is impossible because of the finiteness of computer memory. So, instead, we establish a set $Q^{r e p r}$ which contains as many rational numbers as are possible to represent in the memory available. Despite this finite representation, there are advantages to this approach over other implementations of spatial database systems including those employing realms and dual grids. First of all, the use of $Q^{\text {repr }}$ takes us beyond the fixed lengths available in existing spatial database systems by using arbitrary precision rational number systems. The set $Q^{r e p r}$ of representable rational numbers has much greater precision than types such as float, double and longdouble. Secondly, the set $Q^{r e p r}$ contains many more numbers than there are along an axis in a realm. Thus, we can represent many more coordinates 
than a realm can. In effect, we have a very fine grid here in which the number of grid points available to represent the primitives is $\left|Q^{\text {repr }}\right|^{3}$. This is also an improvement over dual grids because of their need, even in 2D, for two grids, a point grid and segment grid. Our approach requires only one grid. Also, we are not aware of anyone who has actually implemented dual grids, not even in 2D. Finally, because we rationally specify our 3D primitives, the realm based and geometric primitive based layers of [8] merge into a single layer here.

As we specify the primitives, we will, as might be expected, give definitions that indicate what they look like. Lack of space, however, prevents us from giving all the operations of the primitives. Thus, we have several priorities in selecting operations to specify. Most importantly, we define operations on primitives needed to define other primitives. Also, we concentrate on operations for the first three primitives, point, segment and facet. This is because the solid operations are not necessary for defining other primitives. Also, there are far too many operations involving solid to fit in this paper. We must concentrate on predicates as opposed to operations that yield another primitive because predicates are more important for defining other primitives. We do, however, define the important intersection operations that yield a primitive. We will give both signatures and semantics for all operations that we define. We will also provide figures for most of the operations involving facets. Figures for operations involving two segments, however, are already available in the literature such as [8].

\subsection{The Primitives point and segment}

First, we define point and segment. We define these two primitives together because operations between them are necessary to define operations involving segments. The primitive point is a point in $3 \mathrm{D}$ space which has representable rational numbers as its elements. We define it as follows.

$$
\text { point }=\left\{(x, y, z) \mid x, y, z \in Q^{\text {repr }}\right\} .
$$

We need the following two predicates on points to define segment. Suppose $p_{1}=\left(x_{1}, y_{1}, z_{1}\right)$ and $p_{2}=\left(x_{2}, y_{2}, z_{2}\right)$ are two points. When the two points are equal,

$$
\begin{aligned}
& =: \quad \text { point } \times \text { point } \rightarrow \text { bool } \\
& p_{1}=p_{2} \Leftrightarrow x_{1}=x_{2} \wedge y_{1}=y_{2} \wedge z_{1}=z_{2} .
\end{aligned}
$$

When they are not equal,

$$
\begin{aligned}
& \neq: \quad \text { point } \times \text { point } \rightarrow \text { bool } \\
& p_{1} \neq p_{2} \Leftrightarrow x_{1} \neq x_{2} \vee y_{1} \neq y_{2} \vee z_{1} \neq z_{2} .
\end{aligned}
$$

When $p_{1} \neq p_{2}$, we also say that $p_{1}$ and $p_{2}$ are disjoint.

Now, we can define segment. It is an ordered pair of points.

$$
\text { segment }=\left\{\left(p_{1}, p_{2}\right) \mid p_{1}, p_{2} \in \text { point }, p_{1}<p_{2}\right\} .
$$

Thus, $p_{1}$ and $p_{2}$ are the endpoints of a three-dimensional segment. Next, we define two predicates between point and segment that are important in defining operations between two segments. Suppose $p$ is a point and $s=\left(p_{1}, p_{2}\right)$ is a segment where $p_{1}=\left(x_{1}, y_{1}, z_{1}\right)$ and $p_{2}=\left(x_{2}, y_{2}, z_{2}\right)$. Then we say that $p$ on $s$ if $p$ lies on the segment $s$. That is,

$$
\begin{aligned}
& \text { on: point } \times \text { segment } \rightarrow \text { bool } \\
& \text { pon } s \Leftrightarrow p \in\{q=(x, y, z) \mid \\
& \text { (i) }\left(x_{1} \leq x \leq x_{2}\right) \vee\left(x_{2} \leq x \leq x_{1}\right) \\
& \quad \text { (ii) }\left(y_{1} \leq y \leq y_{2}\right) \vee\left(y_{2} \leq y \leq y_{1}\right) \\
& \quad \text { (iii) }\left(z_{1} \leq z \leq z_{2}\right) \vee\left(z_{2} \leq z \leq z_{1}\right) \\
& \text { (iv) } \left.\frac{x-x_{1}}{x_{2}-x_{1}}=\frac{y-y_{1}}{y_{2}-y_{1}}=\frac{z-z_{1}}{z_{2}-z_{1}}\right\}
\end{aligned}
$$

The in predicate is almost the same as the on predicate. The only difference is that the point cannot lie on the endpoints of the segment. Thus,

$$
\begin{aligned}
& \text { in: point } \times \text { segment } \rightarrow \text { bool } \\
& \text { pin } s \Leftrightarrow p \in\{q=(x, y, z) \mid \\
& \text { (i) } q \text { on } s \\
& \text { (ii) }\left(x \neq x_{1}\right) \wedge\left(x \neq x_{2}\right) \\
& \text { (iii) }\left(y \neq y_{1}\right) \wedge\left(y \neq y_{2}\right) \\
& \text { (iv) } \left.\left(z \neq z_{1}\right) \wedge\left(z \neq z_{2}\right)\right\} .
\end{aligned}
$$

When $p$ in $s$, we can also say that $s$ overlaps $p$ or that $s$ intersects $p$. Also, when $\neg(p$ on $s)$, we say that $p$ and $s$ are disjoint.

Now, we define operations between two segments. We refer the reader to [8] for a figure illustrating them. Suppose $s_{1}=\left(p_{1}, p_{2}\right)$ and $s_{2}=\left(q_{1}, q_{2}\right)$ are two segments. Also, suppose $p_{1}=\left(x_{1}, y_{1}, z_{1}\right)$, $p_{2}=\left(x_{2}, y_{2}, z_{2}\right), q_{1}=\left(x_{3}, y_{3}, z_{3}\right)$ and $q_{2}=\left(x_{4}, y_{4}, z_{4}\right)$. When $s_{1}$ and $s_{2}$ are equal,

$$
\begin{aligned}
& =: \quad \text { segment } \times \text { segment } \rightarrow \text { bool } \\
& s_{1}=s_{2} \Leftrightarrow p_{1}=q_{1} \wedge p_{2}=q_{2} .
\end{aligned}
$$

When they are not equal,

$$
\begin{aligned}
& \neq: \quad \text { segment } \times \text { segment } \rightarrow \text { bool } \\
& s_{1} \neq s_{2} \Leftrightarrow p_{1} \neq q_{1} \vee p_{2} \neq q_{2} .
\end{aligned}
$$

The two segments $s_{1}$ and $s_{2}$ overlap if they share a common segment. This means that the segments must lie in the same line. Thus,

$$
\begin{aligned}
& \text { overlaps : } \quad \text { segment } \times \text { segment } \rightarrow \text { bool } \\
& s_{1} \text { overlaps } s_{2} \Leftrightarrow \\
& \text { (i) }\left(p_{1} \text { in } s_{2}\right) \vee\left(p_{2} \text { in } s_{2}\right) \\
& \vee\left(q_{1} \text { in } s_{1}\right) \vee\left(q_{2} \text { in } s_{1}\right) \wedge \\
& \text { (ii) } \frac{x_{3}-x_{1}}{x_{2}-x_{1}}=\frac{y_{3}-y_{1}}{y_{2}-y_{1}}=\frac{z_{3}-z_{1}}{z_{2}-z_{1}} \wedge \\
& \text { (iii) } \frac{x_{4}-x_{1}}{x_{2}-x_{1}}=\frac{y_{4}-y_{1}}{y_{2}-y_{1}}=\frac{z_{4}-z_{1}}{z_{2}-z_{1}} \text {. }
\end{aligned}
$$

They meet if they have exactly one common endpoint and no other common points. Therefore, if the two segments meet, it is not possible for them to intersect at any other place besides their common endpoint. That is, if $\oplus$ is the XOR operator,

$$
\begin{aligned}
& \text { meets: segment } \times \text { segment } \rightarrow \text { bool } \\
& s_{1} \text { meets } s_{2} \Leftrightarrow\left(\text { i) } \neg\left(s_{1} \text { overlaps } s_{2}\right) \wedge\right. \\
& \text { (ii) }\left(p_{1}=q_{1}\right) \oplus\left(p_{1}=q_{2}\right) \\
& \quad \oplus\left(p_{2}=q_{1}\right) \oplus\left(p_{2}=q_{2}\right) .
\end{aligned}
$$

They intersect if they have one common point other than their endpoints. In the next section (Section 4) we show that this common point is rational. More precisely,

$$
\begin{array}{r}
\text { intersects : } \quad \text { segment } \times \text { segment } \rightarrow \text { bool } \\
s_{1} \text { intersects } s_{2} \Leftrightarrow \exists p \in \text { point }:\left(p \text { in } s_{1}\right) \\
\wedge\left(p \text { in } s_{2}\right) .
\end{array}
$$

One segment touches the other segment if they have one common point which is the endpoint of exactly one of the segments.

$$
\begin{aligned}
& \text { touches : segment } \times \text { segment } \rightarrow \text { bool } \\
& s_{1} \text { touches } s_{2} \Leftrightarrow \text { (i) } \neg\left(s_{1} \text { overlaps } s_{2}\right) \wedge \\
& \text { (ii) }\left(p_{1} \text { in } s_{2}\right) \vee\left(p_{2} \text { in } s_{2}\right) \\
& \vee\left(q_{1} \text { in } s_{1}\right) \vee\left(q_{2} \text { in } s_{1}\right) .
\end{aligned}
$$


They are collinear if they are in the same line, but have no common points.

$$
\begin{aligned}
& \text { collinear : } \quad \text { segment } \times \text { segment } \rightarrow \text { bool } \\
& s_{1} \text { collinear } s_{2} \Leftrightarrow \\
& \text { (i) } s_{1} \neq s_{2} \wedge \neg\left(s_{1} \text { meets } s_{2}\right) \\
& \wedge \neg\left(s_{1} \text { overlaps } s_{2}\right) \wedge \\
& \text { (ii) } \frac{x_{3}-x_{1}}{x_{2}-x_{1}}=\frac{y_{3}-y_{1}}{y_{2}-y_{1}}=\frac{z_{3}-z_{1}}{z_{2}-z_{1}} \wedge \\
& \text { (iii) } \frac{x_{4}-x_{1}}{x_{2}-x_{1}}=\frac{y_{4}-y_{1}}{y_{2}-y_{1}}=\frac{z_{4}-z_{1}}{z_{2}-z_{1}} \text {. }
\end{aligned}
$$

The two segments are coplanar if they lie in the same plane. Note that if two segments are not coplanar, then it is impossible for them to meet, intersect, overlap or touch. Neither can they be collinear.

$$
\begin{aligned}
& \text { coplanar: segment } \times \text { segment } \rightarrow \text { bool } \\
& s_{1} \text { coplanar } s_{2} \Leftrightarrow\left|\begin{array}{lll}
x_{2}-x_{1} & y_{2}-y_{1} & z_{2}-z_{1} \\
x_{3}-x_{1} & y_{3}-y_{1} & z_{3}-z_{1} \\
x_{4}-x_{1} & y_{4}-y_{1} & z_{4}-z_{1}
\end{array}\right|=0 .
\end{aligned}
$$

Two segments are disjoint if they have no common points. Stated in terms of the previous definitions, they are disjoint if they are not equal and they do not meet, intersect, overlap or touch.

There is also an operation involving segments that yields another primitive as its result rather than a boolean value. It is intersection. When two segments intersect, the intersection operation yields the point at which they intersect. An intersection may occur whenever the segments meet, intersect or touch. If the segments $s_{1}$ and $s_{2}$ meet, intersect or touch, then

$$
\begin{aligned}
& \text { intersection: } \quad \text { segment } \times \text { segment } \rightarrow \text { point } \\
& \text { intersection }\left(s_{1}, s_{2}\right)=\left(x_{1}+l_{1} t, y_{1}+m_{1} t, z_{1}+n_{1} t\right)
\end{aligned}
$$

where

$$
\begin{aligned}
& t=\frac{l_{3}-m_{3}}{-l_{1} m_{3}+l_{3} m_{1}}, \quad l_{1}=\frac{x_{2}-x_{1}}{d_{1}}, \\
& m_{1}=\frac{y_{2}-y_{1}}{d_{1}}, \quad n_{1}=\frac{z_{2}-z_{1}}{d_{1}}, \\
& d_{1}=\sqrt{\left(x_{2}-x_{1}\right)^{2}+\left(y_{2}-y_{1}\right)^{2}+\left(z_{2}-z_{1}\right)^{2}}, \\
& l_{3}=\frac{x_{4}-x_{3}}{d_{3}}, \quad m_{3}=\frac{y_{4}-y_{3}}{d_{3}}, \quad \text { and } \\
& d_{3}=\sqrt{\left(x_{4}-x_{3}\right)^{2}+\left(y_{4}-y_{3}\right)^{2}+\left(z_{4}-z_{3}\right)^{2}} .
\end{aligned}
$$

We will show that this intersection is rational in the next section (Section 4) despite the presence of square roots.

\subsection{The Primitive facet}

Now we can turn our attention to defining facet. Intuitively, a facet is a finite, contiguous part of a plane in three-dimensional space. We use facets as the discrete building blocks for surfaces, reliefs and boundaries of volumes. To define facet we use segments just like we used points to define segment. That is, segments give the boundary of a facet like points give the boundary or endpoints of a segment. We will refer to the segments forming the boundary of a facet as boundary segments. Rigorously,

$$
\begin{aligned}
& \text { facet }=\left\{\left\{s_{0}, s_{1}, \ldots, s_{m-1}\right\} \mid\right. \\
& \text { (i) } \forall i \in\{0, \ldots, m-1\}, s_{i} \in \text { segment } \\
& \text { (ii) } \forall i \in\{0, \ldots, m-1\}, \\
& s_{i} \text { meets } s_{(i+1)} \bmod m \\
& \text { (iii) No more than two of } s_{0}, s_{1}, \ldots, \text { and } \\
& s_{m-1} \text { meet } \text { at any one point } \\
& \text { (iv) } \forall i, j \in\{0, \ldots, m-1\}, \\
&\left.s_{i} \text { and } s_{j} \text { is coplanar }\right\} .
\end{aligned}
$$

Thus, the boundary segments of a facet are $s_{0}, s_{1}, \ldots, s_{m-1}$.

To define some of the operations involving facets, we need to know whether two objects are in the same plane. So, the first operation we define for facets is the coplanar predicate. We will use the type variable $\gamma$ ranging over \{point, segment, facet $\}$. Let $o \in \gamma$ and let $f$ be a facet. Furthermore, let $p=(x, y, z)$ be a point of $o$ and let $p_{3}=\left(x_{3}, y_{3}, z_{3}\right), p_{4}=\left(x_{4}, y_{4}, z_{4}\right)$ and $p_{5}=\left(x_{5}, y_{5}, z_{5}\right)$ be disjoint points (i.e., endpoints) in the boundary segments of $f$. Then,

$$
\begin{array}{ccc}
\text { coplanar: } \quad \gamma \times \text { facet } \rightarrow \text { bool } \\
\text { o coplanar } f \Leftrightarrow\left|\begin{array}{ccc}
x-x_{3} & y-y_{3} & z-z_{3} \\
x_{4}-x_{3} & y_{4}-y_{3} & z_{4}-z_{3} \\
x_{5}-x_{3} & y_{5}-y_{3} & z_{5}-z_{3}
\end{array}\right|=0 .
\end{array}
$$

More generally, to define operations involving facets, we must consider operations that involve a point, a segment and another facet. We first consider predicates involving a point and a facet. These are the predicates on, in and out. They test whether a point is on one of the boundary segments of a facet, or is inside or outside of the boundary formed by the boundary segments. The on and in operations, in particular, are superficially analogous to the on and in operations for points and segments. If $f$ is a facet, as before, and $p=(x, y, z)$ is a point,

$$
\begin{aligned}
& \text { on: point } \times \text { facet } \rightarrow \text { bool } \\
& \text { pon } f \Leftrightarrow \exists s \in f: \text { pon } s .
\end{aligned}
$$

Suppose $x_{\max }, y_{\max }$ and $z_{\max }$ are the maximum coordinate values in the boundary segments defining the facet $f$. Furthermore, let $p_{\text {big }}=$ $\left(x_{\text {big }}, y_{\text {big }}, z_{\text {big }}\right)$ be a point that is coplanar with the facet $f$ such that $x_{\text {big }}>x_{\max }, y_{b i g}>y_{\max }$ and $z_{\text {big }}>z_{\max }$. Thus, $p_{\text {big }}$ must be outside the facet $f$. Next, let $s_{p}$ be the segment $\left(p, p_{b i g}\right)$. Intuitively, if $p$ is inside the boundary formed by the boundary segments of $f$, then $s_{p}$ must intersect, touch or meet an odd number of the boundary segments. Formally, let $S_{o n}(f)$ be the set of segments in $f$ whose right (but not left) endpoint is on $s_{p}$. Also, let $S_{i}(f)$ be the set of segments in $f$ that intersect $s_{p}$. Then,

$$
\begin{aligned}
& \text { in }: \quad \text { point } \times \text { facet } \rightarrow \text { bool } \\
& \text { pin } f \Leftrightarrow \neg(p \text { on } f) \wedge\left|S_{\text {on }}(f)\right|+\left|S_{i}(f)\right| \text { is odd, } \\
& \text { out }: \quad \text { point } \times \text { facet } \rightarrow \text { bool } \\
& \text { pout } f \Leftrightarrow \neg(p \text { on } f \vee p \text { in } f) .
\end{aligned}
$$

When $p$ out $f$, we also call $p$ and $f$ disjoint.

There are many operations involving a segment and a facet. Figure 1 illustrates them. Let us again suppose that $s=\left(p_{1}, p_{2}\right)$ is a segment and that $f=\left\{s_{0}, s_{1}, \ldots, s_{m-1}\right\}$ is a facet. The segment overlaps the facet when part of it lies inside the facet. That is,

$$
\begin{aligned}
& \text { overlaps: segment } \times \text { facet } \rightarrow \text { bool } \\
& \text { soverlaps } f \Leftrightarrow \text { (i) } s \text { coplanar } f \\
& \text { (ii) }\left(\left(p_{1} \text { in } f \wedge p_{2} \text { out } f\right)\right. \\
& \left.\vee\left(p_{2} \text { in } f \wedge p_{1} \text { out } f\right)\right) \\
& \vee\left(p_{1} \text { out } f \wedge p_{2} \text { out } f\right. \\
& \wedge\left(\exists s_{i} \in f: s_{i} \text { intersects } s\right. \\
& \left.\left.\quad \vee s_{i} \text { touches } s\right)\right) .
\end{aligned}
$$


It meets the facet when it touches or meets a boundary segment of the facet. If the segment touches the facet, however, then its endpoint must lie on the boundary segment of the facet rather than the other way around. (This distinguishes meets from a predicate we will cover later, tangent.)

$$
\begin{aligned}
& \text { meets : segment } \times \text { facet } \rightarrow \text { bool } \\
& \text { s meets } f \Leftrightarrow \\
& \text { (i) } \neg(\text { s overlaps } f) \wedge \\
& \text { (ii) } \exists i \in\{0, \ldots, m-1\}, \exists s_{i} \in f: \\
& \quad s_{i} \text { meets } s \\
& \quad \vee\left(s_{i} \text { touches } s\right. \\
& \\
& \left.\quad \wedge\left(p_{1} \text { on } s_{i} \vee p_{2} \text { on } s_{i}\right)\right) .
\end{aligned}
$$

They intersect if the interior of the segment goes through one point in the interior of the facet. In the next section (Section 4), we show that the point where they intersect is rational and, thus, is a point.

$$
\begin{aligned}
& \text { intersects: } \quad \text { segment } \times \text { facet } \rightarrow \text { bool } \\
& \text { s intersects } f \Leftrightarrow \quad \exists \text { in } s: p \text { in } f \\
& \wedge \neg(\text { coplanar } f) .
\end{aligned}
$$

The segment touches the facet when an endpoint of the segment is in the interior of the facet.

$$
\begin{aligned}
& \text { touches: } \quad \text { segment } \times \text { facet } \rightarrow \text { bool } \\
& \text { stouches } f \Leftrightarrow \quad\left(p_{1} \text { in } f \oplus p_{2} \text { in } f\right) \\
& \wedge \neg(\text { s overlaps } f) .
\end{aligned}
$$

When a segment overlaps a boundary segment of a facet, the segment and facet are incident.

$$
\begin{gathered}
\text { incident : } \quad \text { segment } \times \text { facet } \rightarrow \text { bool } \\
\text { sincident } f \Leftrightarrow \quad \exists i \in\{0, \ldots, m-1\}, \exists s_{i} \in f: \\
\quad s_{i} \text { overlaps } s .
\end{gathered}
$$

They are tangent when the segment intersects one of the boundary segments of the facet. It can also touch the boundary segment, but only if the endpoint of the boundary segment is on the segment.

$$
\begin{aligned}
& \text { tangent : segment } \times \text { facet } \rightarrow \text { bool } \\
& \text { stangent } f \Leftrightarrow \text { (i) } \neg(\text { s overlaps } f) \wedge \\
& \text { (ii) } \exists i \in\{0, \ldots, m-1\}, \exists s_{i} \in f: \\
& \quad\left(s_{i} \text { intersects } s\right) \\
& \vee\left(s_{i} \text { touches } s \wedge \neg p_{1} \text { on } s_{i}\right. \\
& \left.\wedge \neg p_{2} \text { on } s_{i}\right) .
\end{aligned}
$$

Note that a segment can be tangent with a facet at more than one point. The segment lies inside a facet when it is completely within the facet.

$$
\begin{aligned}
& \text { inside: } \quad \text { segment } \\
& \text { sinside } f \Leftrightarrow \text { facet } \rightarrow \text { bool } \\
& \begin{aligned}
& \text { (i) }\left(p_{1} \text { in } f \vee p_{1} \text { on } f\right) \\
& \wedge\left(p_{2} \text { in } f \vee p_{2} \text { on } f\right) \wedge \\
& \text { (ii) } \neg\left(\exists s_{i} \in f: s_{i} \text { intersects } s\right. \\
&\left.\vee s_{i} \text { touches } s\right) .
\end{aligned}
\end{aligned}
$$

It lies outside the facet when none of the segment is in the facet.

$$
\begin{aligned}
& \text { outside }: \quad \text { segment } \times \text { facet } \rightarrow \text { bool } \\
& \text { soutside } f \Leftrightarrow \text { (i) } p_{1} \text { out } f \wedge p_{2} \text { out } f \wedge \\
& \text { (ii) } \neg(\text { s intersects } f) \\
& \wedge \neg(\text { s overlaps } f) \\
& \wedge \neg(\text { s tangent } f) .
\end{aligned}
$$
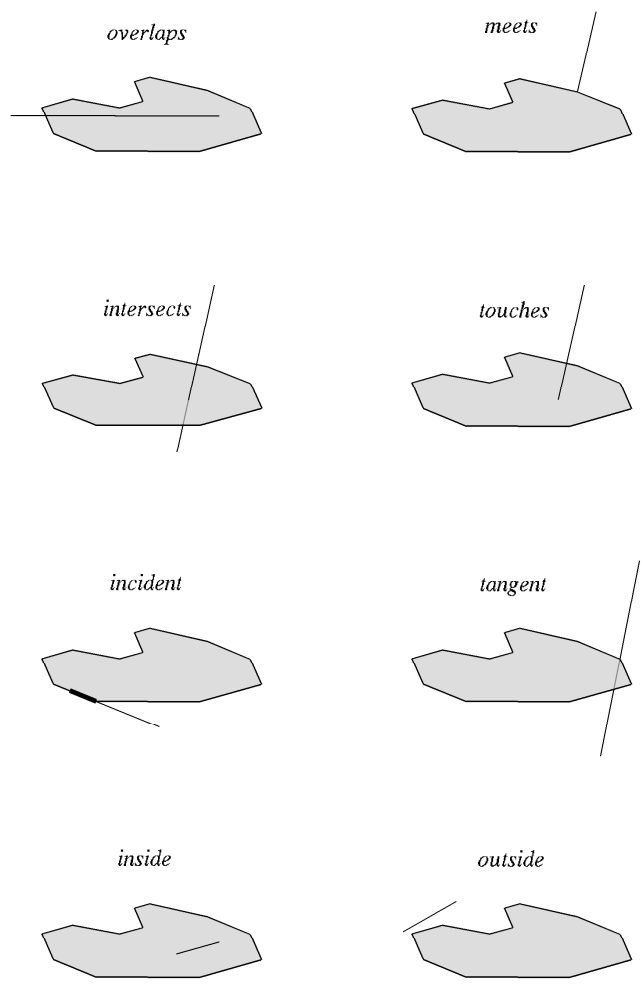

Figure 1: Predicates between a segment and a facet

Equivalently, we say that $s$ and $f$ are disjoint when $s$ outside $f$.

Next, we define an operation involving a segment and a facet that yields another primitive as its result. It is the intersection operation for a segment and a facet. If the segment and facet are not coplanar and meet, intersect, touch or are tangent,

$$
\begin{aligned}
& \text { intersection: } \quad \text { segment } \times \text { facet } \rightarrow \text { point } \\
& \text { intersection }(s, f)=\left(x_{1}+l t, y_{1}+m t, z_{1}+n t\right) \\
& \text { where } \\
& t=\frac{\Gamma\left(x_{3}-x_{1}\right)+\Delta\left(y_{3}-y_{1}\right)+\Phi\left(z_{3}-z_{1}\right)}{\Gamma l+\Delta m+\Phi n}, \\
& l=\frac{x_{2}-x_{1}}{d}, \quad m=\frac{y_{2}-y_{1}}{d}, \quad n=\frac{z_{2}-z_{1}}{d}, \\
& d=\sqrt{\left(x_{2}-x_{1}\right)^{2}+\left(y_{2}-y_{1}\right)^{2}+\left(z_{2}-z_{1}\right)^{2}}, \\
& \Gamma=\left|\begin{array}{ll}
y_{4}-y_{3} & z_{4}-z_{3} \\
y_{5}-y_{3} & z_{5}-z_{3}
\end{array}\right|, \\
& \Delta=\left|\begin{array}{ll}
x_{4}-x_{3} & z_{4}-z_{3} \\
x_{5}-x_{3} & z_{5}-z_{3}
\end{array}\right| \text {, } \\
& \text { and } \Phi=\left|\begin{array}{ll}
x_{4}-x_{3} & y_{4}-y_{3} \\
x_{5}-x_{3} & y_{5}-y_{3}
\end{array}\right| .
\end{aligned}
$$

$\Gamma, \Delta$ and $\Phi$ are the coefficients in the general equation of the plane in which the facet is located. We will show more about how a solution such as this is obtained and that it is rational in Section 4. 
There are also many operations involving two facets. Figure 2 illustrates them. The first four predicates we define are crucial to defining the 3D geometric primitive, solid. They are overlaps, meets, intersects and touches. Let $f_{1}=\left\{s_{10}, s_{11}, \ldots, s_{1, m-1}\right\}$ and $f_{2}=\left\{s_{20}, s_{21}, \ldots, s_{2, l-1}\right\}$ be two facets where $s_{1 j}=\left(p_{1 j 1}, p_{1 j 2}\right) \forall j$ $\in\{0, \ldots, m-1\}$ and $s_{2 j}=\left(p_{2 j 1}, p_{2 j 2}\right) \forall j \in\{0, \ldots, l-1\}$. The overlaps operation between two facets indicates whether part of their interiors coincide. Thus, two facets overlap when they are coplanar and a point (i.e., an endpoint) of at least one boundary segment of one of the facets is in the other facet. Thus,

$$
\begin{aligned}
& \text { overlaps : facet } \times \text { facet } \rightarrow \text { bool } \\
& f_{1} \text { overlaps } f_{2} \Leftrightarrow \text { (i) } f_{1} \text { coplanar } f_{2} \wedge \\
& \text { (ii) } \exists s_{1 j} \in f_{1}:\left(p_{1 j 1} \text { in } f_{2}\right) \\
& \vee\left(p_{1 j 2} \text { in } f_{2}\right) \\
& \vee \exists s_{2 j} \in f_{2}:\left(p_{2 j 1} \text { in } f_{1}\right) \\
& \vee\left(p_{2 j 2} \text { in } f_{1}\right) \text {. }
\end{aligned}
$$

The meets operation for facets will be very important in defining solid. Two facets meet if they have boundary segments which are equal. Equivalently, we could say that they have at least one common segment. When two facets meet, the two boundary segments must be equal and not merely overlap. Furthermore, they have nothing else in common besides segments that are equal. We will handle the case of when the two boundary segments overlap later in a different predicate called incident. This makes our definition of meets more restricted than the predicate usually called meets in the 9-intersection model. We find this restriction necessary in order to define solid.

$$
\begin{aligned}
& \text { meets : facet } \times \text { facet } \rightarrow \text { bool } \\
& f_{1} \text { meets } f_{2} \Leftrightarrow \text { (i) } \neg\left(f_{1} \text { overlaps } f_{2}\right) \\
& \wedge \neg\left(f_{2} \text { overlaps } f_{1}\right) \wedge \\
& \text { (ii) } \exists s_{1 i} \in f_{1}, \exists s_{2 j} \in f_{2} \text { : } \\
& s_{1 i}=s_{2 j} \wedge \\
& \text { (iii) } \neg\left(\exists s_{1 i} \in f_{1}, \exists s_{2 j} \in f_{2}\right. \text { : } \\
& s_{1 i} \text { overlaps } s_{2 j} \\
& \wedge s_{1 i} \text { intersects } s_{2 j} \\
& \wedge s_{1 i} \text { touches } s_{2 j} \text { ). }
\end{aligned}
$$

The facets intersect if they have at least one common segment in their interiors. Therefore, facets that intersect cannot be coplanar. It is correct to refer to their intersection as containing a segment because segment is rationally defined as we show in Section 4 .

$$
\begin{aligned}
& \text { intersects : facet } \times \text { facet } \rightarrow \text { bool } \\
& f_{1} \text { intersects } f_{2} \Leftrightarrow\left(\exists s_{1 j} \in f_{1}: s_{1 j} \text { intersects } f_{2}\right) \\
& \vee\left(\exists s_{2 j} \in f_{2}: s_{2 j} \text { intersects } f_{1}\right) \\
& \vee\left(\left(\exists s_{1 j} \in f_{1}: s_{1 j} \text { tangent } f_{2}\right)\right. \\
& \left.\wedge\left(\exists s_{2 j} \in f_{2}: s_{2 j} \text { tangent } f_{1}\right)\right)
\end{aligned}
$$

One facet touches another when a single point of one of the facets is in the interior of the other facet. This can only happen when a point (i.e., an endpoint) of a boundary segment of one of the facets is in the other facet. The meets predicate does not allow an entire boundary segment to be inside the other facet. The brushes predicate, which we will define later, allows a boundary segment of one facet to be inside the other facet.

$$
\begin{aligned}
& \text { touches: facet } \times \text { facet } \rightarrow \text { bool } \\
& f_{1} \text { touches } f_{2} \Leftrightarrow \\
& \text { (i) } \neg\left(f_{1} \text { overlaps } f_{2}\right) \\
& \wedge \neg\left(f_{1} \text { intersects } f_{2}\right) \wedge \\
& \text { (ii) }\left(\exists s_{1 i} \in f_{1}: s_{1 i} \text { touches } f_{2}\right) \\
& \vee\left(\exists s_{2 i} \in f_{2}: s_{2 i} \text { touches } f_{1}\right) \text {. }
\end{aligned}
$$
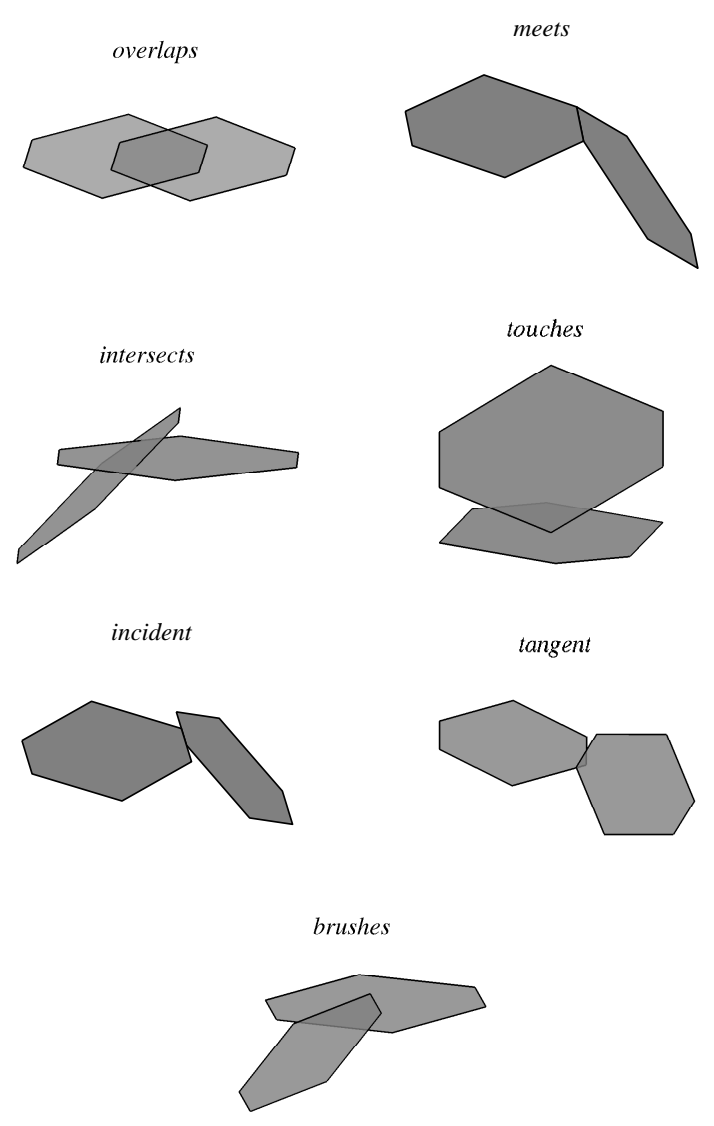

Figure 2: Predicates between two facets

They are incident when a boundary segment from both overlap.

$$
\begin{aligned}
& \text { incident }: \quad \text { facet } \times \text { facet } \rightarrow \text { bool } \\
& f_{1} \text { incident } f_{2} \Leftrightarrow \\
& \text { (i) } \neg\left(f_{1} \text { overlaps } f_{2}\right) \wedge \\
& \text { (ii) }\left(\exists s_{1 i} \in f_{1}: s_{1 i} \text { incident } f_{2}\right) \\
& \quad \vee\left(\exists s_{2 i} \in f_{2}: s_{2 i} \text { incident } f_{1}\right) .
\end{aligned}
$$

They are tangent if their boundaries have a common point.

$$
\begin{aligned}
& \text { tangent : facet } \times \text { facet } \rightarrow \text { bool } \\
& f_{1} \text { tangent } f_{2} \Leftrightarrow \\
& \text { (i) } \neg\left(f_{1} \text { overlaps } f_{2}\right) \\
& \wedge \neg\left(f_{1} \text { intersects } f_{2}\right) \wedge \\
& \text { (ii) }\left(\exists s_{1 i} \in f_{1}: s_{1 i} \text { tangent } f_{2}\right) \\
& \vee\left(\exists s_{2 i} \in f_{2}: s_{2 i} \text { tangent } f_{1}\right) \text {. }
\end{aligned}
$$

The two facets brush if part of one or more segments of one facet lie in the interior of the other facet when the facets are not coplanar.

$$
\begin{aligned}
& \text { brushes: } \quad \text { facet } \times \text { facet } \rightarrow \text { bool } \\
& f_{1} \text { brushes } f_{2} \Leftrightarrow \text { (i) } \neg\left(f_{1} \text { coplanar } f_{2}\right) \wedge \neg\left(f_{1} \text { intersects } f_{2}\right) \wedge \\
& \text { (ii) }\left(\exists s_{1 i} \in f_{1}: s_{1 i} \text { inside } f_{2}\right. \\
&\left.\vee s_{1 i} \text { overlaps } f_{2}\right) \\
& \vee\left(\exists s_{2 i} \in f_{2}: s_{2 i} \text { inside } f_{1}\right. \\
&\left.\quad \vee s_{2 i} \text { overlaps } f_{1}\right) .
\end{aligned}
$$


Moreover, two facets are disjoint if they have no points in common. That is, when they are disjoint, they do not overlap, meet, intersect, touch or brush or are not incident or tangent.

Finally, we define the intersection operation for two facets. It yields points and segments as its result. Its signature indicates this:

$$
\text { intersection: } \begin{aligned}
\text { facet } & \times \text { facet } \\
& \rightarrow\{s \mid s \in \text { point } \cup \text { segment }\} .
\end{aligned}
$$

The intersection operation is critical for the definition of solid. To define the intersection of two facets we determine the point set of the line formed by the intersection of the supporting planes of the two facets and confine the point set to the facets themselves. In doing so, we use the intersection operation for a segment and a facet. The result is a set of points and segments that are all collinear. Suppose $s_{1 i}=\left(p_{1 i 1}, p_{1 i 2}\right)$ and $s_{1 j}=\left(p_{1 j 1}, p_{1 j 2}\right)$ are boundary segments of facet $f_{1}$ such that $p_{1 i 1}=\left(x_{3}, y_{3}, z_{3}\right), p_{1 i 2}=\left(x_{4}, y_{4}, z_{4}\right)$ and $p_{1 j 1}=\left(x_{5}, y_{5}, z_{5}\right)$. Suppose further that $s_{2 i}=\left(p_{2 i 1}, p_{2 i 2}\right)$ and $s_{2 j}=$ $\left(p_{2 j 1}, p_{2 j 2}\right)$ are boundary segments of facet $f_{2}$ such that $p_{2 i 1}=$ $\left(x_{6}, y_{6}, z_{6}\right), p_{2 i 2}=\left(x_{7}, y_{7}, z_{7}\right)$ and $p_{2 j 1}=\left(x_{8}, y_{8}, z_{8}\right)$. Assume that the two facets are not coplanar.

To give the semantics of this operation, we start by defining a function that gives the point set for the line formed by the supporting planes of the facets. We will need this function when we put into lexicographic order the boundary points of segments in the intersection. In the equations that define this point set, $\Gamma 1, \Delta 1, \Phi 1$, $\Psi 1, \Gamma 2, \Delta 2, \Phi 2$ and $\Psi 2$ are the coefficients and constant terms in the general equations of the two supporting planes.

$$
\begin{aligned}
& \text { line }: \quad \text { facet } \times \text { facet } \rightarrow\{p \mid p \in \text { point }\} \\
& \text { line }\left(f_{1}, f_{2}\right)=\left\{\begin{aligned}
\{p & =(x, y, z) \mid \forall t \in Q^{\text {repr }}, \\
& \left.x=x_{0}+l t, y=y_{0}+m t, z=z_{0}+n t\right\}
\end{aligned}\right.
\end{aligned}
$$

where

$$
\begin{aligned}
& l=\left|\begin{array}{ll}
\Delta_{1} & \Phi_{1} \\
\Delta_{2} & \Phi_{2}
\end{array}\right|, \quad m=\left|\begin{array}{ll}
\Phi_{1} & \Gamma_{1} \\
\Phi_{2} & \Gamma_{2}
\end{array}\right|, \\
& n=\left|\begin{array}{ll}
\Gamma_{1} & \Delta_{1} \\
\Gamma_{2} & \Delta_{2}
\end{array}\right|, \\
& x_{0}=\frac{m\left|\begin{array}{ll}
\Psi_{1} & \Phi_{1} \\
\Psi_{2} & \Phi_{2}
\end{array}\right|-n\left|\begin{array}{ll}
\Psi_{1} & \Delta_{1} \\
\Psi_{2} & \Delta_{2}
\end{array}\right|}{l^{2}+m^{2}+n^{2}}, \\
& y_{0}=\frac{n\left|\begin{array}{ll}
\Psi_{1} & \Gamma_{1} \\
\Psi_{2} & \Gamma_{2}
\end{array}\right|-l\left|\begin{array}{ll}
\Psi_{1} & \Phi_{1} \\
\Psi_{2} & \Phi_{2}
\end{array}\right|}{l^{2}+m^{2}+n^{2}} \\
& z_{0}=\frac{l\left|\begin{array}{ll}
\Psi_{1} & \Delta_{1} \\
\Psi_{2} & \Delta_{2}
\end{array}\right|-m\left|\begin{array}{ll}
\Psi_{1} & \Gamma_{1} \\
\Psi_{2} & \Gamma_{2}
\end{array}\right|}{l^{2}+m^{2}+n^{2}}, \\
& \Gamma_{1}=\left|\begin{array}{ll}
y_{4}-y_{3} & z_{4}-z_{3} \\
y_{5}-y_{3} & z_{5}-z_{3}
\end{array}\right|, \\
& \Delta_{1}=\left|\begin{array}{ll}
x_{4}-x_{3} & z_{4}-z_{3} \\
x_{5}-x_{3} & z_{5}-z_{3}
\end{array}\right|, \\
& \Phi_{1}=\left|\begin{array}{ll}
x_{4}-x_{3} & y_{4}-y_{3} \\
x_{5}-x_{3} & y_{5}-y_{3}
\end{array}\right|, \\
& \Gamma_{1} x_{3}+\Delta_{1} y_{3}+\Phi_{1} z_{3},
\end{aligned}
$$

$$
\begin{aligned}
& \Gamma_{2}=\left|\begin{array}{ll}
y_{7}-y_{6} & z_{7}-z_{6} \\
y_{8}-y_{6} & z_{8}-z_{6}
\end{array}\right|, \\
& \Delta_{2}=\left|\begin{array}{ll}
x_{7}-x_{6} & z_{7}-z_{6} \\
x_{8}-x_{6} & z_{8}-z_{6}
\end{array}\right|, \\
& \Phi_{2}=\left|\begin{array}{ll}
x_{7}-x_{6} & y_{7}-y_{6} \\
x_{8}-x_{6} & y_{8}-y_{6}
\end{array}\right|, \\
& \text { and } \quad \quad \Psi_{2}=\Gamma_{2} x_{6}+\Delta_{2} y_{6}+\Phi_{2} z_{6} .
\end{aligned}
$$

Next, we get the places where the facets intersect in a single point which we denote as the set $P_{p}$. For this purpose, we define the set $S_{p}$ which is the set of all boundary segments in one of the facets that make contact with the other facet at the places where the facets intersect in a point. Let

$$
\begin{aligned}
& S_{p}=\left\{s \in f_{1} \cup f_{2} \mid\right. \\
& \quad \text { (i) } f_{1} \text { touches } f_{2} \vee f_{1} \text { tangent } f_{2} \\
& \left.\quad \text { (ii) } \exists i \in\{1,2\}: s \text { touches } f_{i} \vee \text { s tangent } f_{i}\right\}
\end{aligned}
$$

and let

$$
\begin{aligned}
& P_{p}=\left\{\text { intersection }\left(s, f_{1}\right) \mid s \in S_{p}\right\} \\
& \cup\left\{\text { intersection }\left(s, f_{2}\right) \mid s \in S_{p}\right\} .
\end{aligned}
$$

We will eventually put the point set $P_{p}$ in the intersection of the two facets. Before we do that, however, we determine the places where the facets intersect in a segment. This is the set $P_{s}$. To do so, we define the set $S_{S}$ which contains all boundary segments in one of the facets that intersect the other facet at the places where the facets intersect in a segment. Let

$$
S_{s}=\left\{s \in f_{1} \cup f_{2} \mid\right.
$$

(i) $f_{1}$ meets $f_{2} \vee f_{1}$ intersects $f_{2}$ $\vee f_{1}$ incident $f_{2} \vee f_{1}$ brushes $f_{2}$

(ii) $\exists i \in\{1,2\}: s$ meets $f_{i} \vee s$ intersects $f_{i}$ $\vee$ s touches $f_{i} \vee$ s incident $f_{i}$ $\vee$ s tangent $\left.f_{i}\right\}$

and let

$$
\begin{aligned}
& P_{s}=\left\{\text { intersection }\left(s, f_{1}\right) \mid s \in S_{s}\right\} \\
& \cup\left\{\text { intersection }\left(s, f_{2}\right) \mid s \in S_{s}\right\} .
\end{aligned}
$$

Lastly, take the points $P_{S}$, reorder them in lexicographic order along the line $\left(f_{1}, f_{2}\right)$. We denote the reordered set as $P_{o}$. It must have an even number of points because all of its points come from places where the boundary of one facet must go through the other facet and, therefore, must come back through the other facet again. Thus,

$$
\begin{aligned}
P_{o}= & \left\{\left\{p_{1}, p_{2}, \ldots, p_{k}\right\} \mid\right. \\
& \left.k \text { is even } \wedge \forall i \in\{1,2, \ldots, k\}: p_{i} \in P_{S}\right\} .
\end{aligned}
$$

Furthermore, every pair of points represents a segment in the intersection of the two facets. Thus, for $P_{o}=\left\{p_{1}, p_{2}, \ldots, p_{k}\right\}$, let

$$
S_{o}=\left\{\left(p_{1}, p_{2}\right),\left(p_{3}, p_{4}\right), \ldots,\left(p_{k-1}, p_{k}\right)\right\} .
$$

$S_{o}$ contains the segments in the intersection of the facets. Thus, recalling that $P_{p}$ is the set of single points in the intersection,

$$
\text { intersection }\left(f_{1}, f_{2}\right)=P_{p} \cup S_{o} .
$$

This definition of intersection insures that the intersection is empty when the two facets do not meet, intersect, touch or brush or are not incident or tangent. 


\subsection{The Primitive solid}

As we begin the discussion of the geometric primitive solid, we emphasize that while the abstract data type volume must provide for holes, solid does not need them. A 3D object with a hole is not a primitive. Rather, we would represent such an object in computer memory by using two primitives.

Just like we used segments to define facet, we now use facets to define solid. The segments that define a facet essentially give the boundary of the facet and we assume the facet to be inside the boundary. The segments are connected in a way that they meet in pairs so that they enclose the area which is the facet. Similarly, we shall define a solid by using connected facets that meet at boundary segments so that they enclose a section of 3D space. Thus, the enclosing facets are the boundary of a solid. Furthermore, the boundary facets and the 3D space enclosed by them are the solid. Note that not just any collection of connected facets defines a solid. If a collection of connected facets does not enclose a section of 3D space, it is simply a surface. For a collection of facets to define a solid, every facet in the collection must meet exactly one other facet in the collection at everyone of its boundary segments. No boundary segment in a facet can fail to be involved in meeting another facet. More formally,

$$
\begin{gathered}
\text { solid }=\left\{\left\{f_{0}, f_{1}, \ldots, f_{n}\right\} \mid\right. \\
\text { (i) } \forall i \in\{0, \ldots, n\}: f_{i} \in \text { facet } \\
\text { with } f_{i}=\left\{s_{i 0}, s_{i 1}, \ldots, s_{i, m-1}\right\} \\
\text { (ii) } \forall i \in\{0, \ldots, n\}, \forall s_{i k} \in f_{i}, \\
\exists j \in\{0, \ldots, n\} \text { with } j \neq i: \\
\left(f_{i} \text { meets } f_{j}\right) \\
\wedge s_{i k} \in \text { intersection }\left(f_{i}, f_{j}\right) \\
\text { (iii) No more than two of } f_{0}, f_{1}, \ldots, \\
\text { and } \left.f_{n} \text { meet at any one segment }\right\} .
\end{gathered}
$$

There is such a large proliferation of operations involving solids that, by themselves, they would fill a large part of this paper. There are operations between points and solids, between segments and solids, between facets and solids and between two solids. Predicates for points and solids are similar to what we have seen between points and the other two primitives: on, in and out. Examples of operations for segments and solids are meets, intersects, touches, incident, tangent, inside and outside (disjoint). Examples of operations for facets and solids are meets, intersects, cuts, touches, incident, tangent, brushes, inside, outside (disjoint), cutabove and cutbelow. Finally, examples of operations involving two solids are equals, not equals, meets, joins, intersects, incident, connects, links, tangent, brushes, inside, contains, disjoint and intersection.

Due to space limitations, however, we cannot include the definitions of operations of solid. For the same reason, we also cannot include the specification of directional predicates such as above, below, rightside, leftside, before and behind.

Thus, the four geometric primitives in 3D are point, segment, facet and solid. The definition of point uses a rational coordinate. Furthermore, we use points to build a segment, segments to build a facet and facets to build a solid. Therefore, all four geometric primitives, point, segment, facet and solid have a rational specification. We are about to show in Section 4 that the four primitives are closed under intersection up to the limits of computer memory. Once we have done so, it paves the way to implement a 3D spatial database system that relies on arbitrary precision rational arithmetic rather than on realms or dual grids. Hence, we completely eliminate the need to use realms or dual grids. In effect, a rational implementation results in an arbitrarily fine grid. This introduces greater exactness of computation to a spatial database system.
Because of the closure problems of extending realms to 3D that we noted in Section 2, comparison of the efficiency of a rational implementation of a 3D system is not crucial when considering its robustness. We still state, however, that we believe that a rational implementation saves execution time when compared with a hypothetical extension of realms to 3D. This is because a rational implementation does not need the redrawing and enveloping process required by realms and dual grids, not to speak of the preand post-processing algorithms required by the enhanced form of dual grids. Most of the operations involving our rationally specified primitives involve only mathematical formulas that require constant time. This benefit is partially offset because we assume that arithmetic will be performed by an arbitrary precision rational number system where the lengths of numbers can vary. We feel, though, that the problems of realms and dual grids that we have previously mentioned more than outweigh the complexities introduced by arbitrary precision rational arithmetic. Therefore, greater robustness results from the rational implementation of geometric primitives.

\section{THE RATIONAL CLOSURE OF INTER- SECTIONS IN THREE DIMENSIONS}

We now proceed to discuss the closure of 3D geometric primitives under intersection operations. We will prove that intersections involving rationally specified segments and facets are rational. Taken together with the closure of rational arithmetic, the closure of intersections means that a closed system results, as far as spatial operations are concerned, up to the limit of the precision with which computer memory can store rational numbers. This provides for a large amount of robustness in a 3D spatial database system. We need not consider other spatial operations such as union, difference and symmetric difference as the proofs of the closures of those operations follow from the closure of intersections.

Note that considering the intersection of a line and a plane or two lines amounts to considering the intersection between a segment and a facet or between two segments. However, the intersection of two facets is different from the intersection of two planes. Thus, the specific intersections we will consider are of a line and a plane, of two lines and of two facets. All other intersections, including all that involve solids, can be easily derived using these three results.

\subsection{The Intersection of a Line and a Plane}

We first prove that the intersection of a rationally specified line and plane in $3 \mathrm{D}$ is rational. We do this first because the proofs involving the other intersections depend on this one. Indeed, once this proof is given, the proofs for the other two intersections follow fairly quickly.

Let $\left(x_{1}, y_{1}, z_{1}\right)$ and $\left(x_{2}, y_{2}, z_{2}\right)$ be rational points defining a line. We will work with the equations of a $3 \mathrm{D}$ line in parametric form. The parametric equations of a line are

$$
x=x_{1}+l t \quad y=y_{1}+m t \quad z=z_{1}+n t
$$

where $l, m, n$ are the direction cosines for the line defined by $\left(x_{1}\right.$, $\left.y_{1}, z_{1}\right)$ and $\left(x_{2}, y_{2}, z_{2}\right)$. The direction cosines are

$$
\begin{gathered}
\quad l=\frac{x_{2}-x_{1}}{d} \quad m=\frac{y_{2}-y_{1}}{d} \quad n=\frac{z_{2}-z_{1}}{d} \\
\text { where } \quad d=\sqrt{\left(x_{2}-x_{1}\right)^{2}+\left(y_{2}-y_{1}\right)^{2}+\left(z_{2}-z_{1}\right)^{2}} .
\end{gathered}
$$

The intercept form of the definition of a plane in $3 \mathrm{D}$ is

$$
\frac{x}{a}+\frac{y}{b}+\frac{z}{c}=1
$$

where $a, b$ and $c$ are the $x, y$ and $z$ intercepts of the plane. 
Now, suppose $\left(x_{0}, y_{0}, z_{0}\right)$ is the point where the line and the plane intersect. Let $t_{0}$ be the $t$ in the parametric equations at the intersection point $\left(x_{0}, y_{0}, z_{0}\right)$. Then, the equations 1 and 4 at the intersection point $\left(x_{0}, y_{0}, z_{0}\right)$ are

$$
\begin{gathered}
x_{0}=x_{1}+l t_{0}=y_{1}+m t_{0} \quad z_{0}=z_{1}+n t_{0} \\
\frac{x_{0}}{a}+\frac{y_{0}}{b}+\frac{z_{0}}{c}=1 .
\end{gathered}
$$

The resulting equations 5 and 6 are a system of four equations in the four variables $x_{0}, y_{0}, z_{0}, t_{0}$ which correspond to the intersection point $\left(x_{0}, y_{0}, z_{0}\right)$. We solve it by substitution starting with plugging the equations 5 into equation 6 .

$$
\begin{gathered}
\frac{x_{1}+l t_{0}}{a}+\frac{y_{1}+m t_{0}}{b}+\frac{z_{1}+n t_{0}}{c}=1 \\
\Rightarrow \quad \frac{l t_{0}}{a}+\frac{m t_{0}}{b}+\frac{n t_{0}}{c}=1-\frac{x_{1}}{a}-\frac{y_{1}}{b}-\frac{z_{1}}{c} \\
\Rightarrow \quad t_{0}\left(\frac{l}{a}+\frac{m}{b}+\frac{n}{c}\right)=1-\frac{x_{1}}{a}-\frac{y_{1}}{b}-\frac{z_{1}}{c} \\
\Rightarrow \quad t_{0}=\frac{1-\frac{x_{1}}{a}-\frac{y_{1}}{b}-\frac{z_{1}}{c}}{\frac{l}{a}+\frac{m}{b}+\frac{n}{c}}
\end{gathered}
$$

Now we can plug the resulting formula for $t_{0}$ back into equations 5 to get the coordinates at the intersection point $\left(x_{0}, y_{0}, z_{0}\right)$.

$$
\begin{aligned}
& x_{0}=x_{1}+l\left(\frac{1-\frac{x_{1}}{a}-\frac{y_{1}}{b}-\frac{z_{1}}{c}}{\frac{l}{a}+\frac{m}{b}+\frac{n}{c}}\right) \\
& y_{0}=y_{1}+m\left(\frac{1-\frac{x_{1}}{a}-\frac{y_{1}}{b}-\frac{z_{1}}{c}}{\frac{l}{a}+\frac{m}{b}+\frac{n}{c}}\right) \\
& z_{0}=z_{1}+n\left(\frac{1-\frac{x_{1}}{a}-\frac{y_{1}}{b}-\frac{z_{1}}{c}}{\frac{l}{a}+\frac{m}{b}+\frac{n}{c}}\right)
\end{aligned}
$$

The only variables that can produce irrational results in the equations 7 to 9 are $l, m, n$, the direction cosines, and $a, b$ and $c$, the $x, y$ and $z$ intercepts of the plane. Everything else in these equations involves coordinates that we assume to be rational.

Let us first look at the direction cosines $l, m, n$ in the equations 7 to 9 . The thing that suggests the possibility of irrational results from the direction cosines are the square roots seen in the equations 2 and 3 that define the direction cosines. However, the following discussion shows that the square roots all cancel each other. We will use $d$ from equation 3 to represent the square root. Observe what happens to the denominator of the equations 7 to 9 .

$$
\begin{aligned}
\frac{l}{a}+\frac{m}{b} & +\frac{n}{c}=\frac{\left(x_{2}-x_{1}\right) / d}{a}+\frac{\left(y_{2}-y_{1}\right) / d}{b}+\frac{\left(z_{2}-z_{1}\right) / d}{c} \\
& =\frac{1}{d}\left(\frac{\left(x_{2}-x_{1}\right)}{a}+\frac{\left(y_{2}-y_{1}\right)}{b}+\frac{\left(z_{2}-z_{1}\right)}{c}\right)
\end{aligned}
$$

Thus, $\frac{1}{d}$ factors out of the denominator of all of the equations for the intersection coordinates (equations 7 to 9). Now, note that there is a direction cosine as a coefficient to the fractions in all these equations. Thus, the numerator in each equation for a coordinate also has a $\frac{1}{d}$ factor. Thus, the two factors cancel each other eliminating the square root $d$ from the intersection coordinate equations. Thus, the direction cosines $l, m, n$ do not introduce an irrational component to the intersection coordinates.

Next, we will show that the $x, y$ and $z$ intercepts $a, b$ and $c$ must be rational. Let $\left(x_{3}, y_{3}, z_{3}\right),\left(x_{4}, y_{4}, z_{4}\right)$ and $\left(x_{5}, y_{5}, z_{5}\right)$ be rational coordinates defining the plane. Then, plugging these coordinates into equation 4 we get

$$
\begin{aligned}
& \frac{x_{3}}{a}+\frac{y_{3}}{b}+\frac{z_{3}}{c}=1 \\
& \frac{x_{4}}{a}+\frac{y_{4}}{b}+\frac{z_{4}}{c}=1 \\
& \frac{x_{5}}{a}+\frac{y_{5}}{b}+\frac{z_{5}}{c}=1 .
\end{aligned}
$$

This is a system of three linear equations for $\frac{1}{a}, \frac{1}{b}$ and $\frac{1}{c}$. Therefore, Cramer's Rule gives as solutions

$$
\begin{gathered}
\frac{1}{a}=\frac{\left|\begin{array}{lll}
1 & y_{3} & z_{3} \\
1 & y_{4} & z_{4} \\
1 & y_{5} & z_{5}
\end{array}\right|}{\left|\begin{array}{lll}
x_{3} & y_{3} & z_{3} \\
x_{4} & y_{4} & z_{4} \\
x_{5} & y_{5} & z_{5}
\end{array}\right|}, \quad \frac{1}{b}=\frac{\mid \begin{array}{l}
x_{3} \\
x_{4} \\
x_{5}
\end{array}}{\mid \begin{array}{l}
x_{3} \\
x_{4} \\
x_{5}
\end{array}} \\
\frac{1}{c}=\frac{\left|\begin{array}{lll}
x_{3} & y_{3} & 1 \\
x_{4} & y_{4} & 1 \\
x_{5} & y_{5} & 1
\end{array}\right|}{\left|\begin{array}{lll}
x_{3} & y_{3} & z_{3} \\
x_{4} & y_{4} & z_{4} \\
x_{5} & y_{5} & z_{5}
\end{array}\right|} .
\end{gathered}
$$$$
\frac{1}{b}=\frac{\left|\begin{array}{lll}
x_{3} & 1 & z_{3} \\
x_{4} & 1 & z_{4} \\
x_{5} & 1 & z_{5}
\end{array}\right|}{\left|\begin{array}{lll}
x_{3} & y_{3} & z_{3} \\
x_{4} & y_{4} & z_{4} \\
x_{5} & y_{5} & z_{5}
\end{array}\right|},
$$

Determinants are completely evaluated using additions, subtractions and multiplications. Also, we use a division of determinants to determine $\frac{1}{a}, \frac{1}{b}$ and $\frac{1}{c}$. Since the rational numbers are closed under arithmetic, equations 10 and 11 show that $a, b$ and $c$ must be rational.

So, we have shown that the direction cosines $l, m, n$ do not introduce an irrational component to the intersection coordinates and that $x, y$ and $z$ intercepts of the plane must be rational. Thus, the equations 7 to 9 give rational coordinates of the intersection. So, a line and plane in 3D defined using rational numbers must have a rational intersection.

\subsection{The Intersection of Two Lines}

Next, we prove that two rationally specified intersecting lines in $3 \mathrm{D}$ have a rational intersection. Suppose we have two intersecting lines in 3D which are defined using rational points. Let $\left(x_{1}, y_{1}, z_{1}\right)$ and $\left(x_{2}, y_{2}, z_{2}\right)$ be the rational points defining one of the lines and let $\left(x_{3}, y_{3}, z_{3}\right)$ and $\left(x_{4}, y_{4}, z_{4}\right)$ be the rational points defining the other line. Furthermore, let $\left(x_{0}, y_{0}, z_{0}\right)$ be the point where they intersect. Choose any other rational point $\left(x_{5}, y_{5}, z_{5}\right)$ that is not coplanar with the two lines. Then, the points $\left(x_{1}, y_{1}, z_{1}\right),\left(x_{2}, y_{2}, z_{2}\right)$ and $\left(x_{5}, y_{5}, z_{5}\right)$ rationally specify a plane. Furthermore, the line defined by $\left(x_{3}, y_{3}, z_{3}\right)$ and $\left(x_{4}, y_{4}, z_{4}\right)$ intersects this plane at $\left(x_{0}, y_{0}, z_{0}\right)$. Both the plane and the line are rationally specified. Thus, by the result of the previous subsection (Subsection 4.1), $\left(x_{0}, y_{0}, z_{0}\right)$ must be rational. This point, however, is the point at which the two lines intersect. Thus, the two lines have a rational intersection. 


\subsection{The Intersection of Two facets}

Finally, we prove that two facets have a rational intersection. First of all, note that both facets involved in such an intersection have rationally defined supporting planes. This is because we defined the endpoints of the segments forming the boundaries of facets to have rational coordinates. A facet must have at least three of these rational endpoints (and, in fact, probably has many more). Thus, the supporting planes are rationally defined.

Secondly, recall that there can be both points and segments in the intersection of two facets. Therefore, when considering the intersection of two facets, there are two cases. There is the case when there are only points in the intersection and the case when the intersection includes segments. When there are only points in the intersection, they must occur at places where the facets touch or are tangent. When an intersection point is produced because the facets touch, it must occur at the endpoint of a rationally defined segment. The endpoints of segments are points and, therefore, rational. When an intersection point is where the two facets are tangent, it must occur where two segments of the facets intersect or touch. In either situation, the previous subsection (Subsection 4.2) shows that the intersection is rational. Thus, the points in the intersection of the facets are rational. Observe that, when there are more than two of these intersection points, they must be on the same line. This line is the intersection of the supporting planes of the two facets. The line of intersection of the supporting planes is therefore rationally defined (i.e., has at least two rational coordinates).

Suppose there are one or more line segments at the intersection of the facets. To show that the intersection is rational amounts to showing that the supporting line of the line segments formed by the intersection of the facets is rationally defined. Two boundary segments of the facets must intersect the supporting line at separate points. Now, suppose both intersecting boundary segments of a facet cross the other facet at an endpoint. These two endpoints are rational. Since they lie on the supporting line of the intersection of the facets, the line is rationally defined.

Let us assume therefore that the intersections do not occur at an endpoint of the segments. Thus, they occur in the interior of the two segments crossing the facets. Both segments and both facets are rationally defined. So, by Subsection 4.1, the points at the intersection of the segments and the supporting planes of the facets are rational. Both of these points, however, are in the supporting line at the intersection of the facets. Thus, the supporting line is rationally defined implying that the facets have a rational intersection. (Now, the situations where one segment intersects a facet at an endpoint and the other in its interior follow from these results.)

\section{CONCLUSIONS AND FUTURE WORK}

In this paper, we have given a design of robust three-dimensional geometric primitives. Their robustness comes from the use of rational numbers to specify them. We have compared our design for the primitives with extensions from two dimensions of simplicial complexes, realms and dual grids (near the beginning and at the end of Section 3). This has included reviewing the potential of rational arithmetic and discussing the amount of representable numbers, time requirements and the rational closure of intersections of the primitives.

In the future, we need to complete the definitions of the operations for the geometric primitives, especially those involving solid, those that yield another primitive as their result and those that are directional predicates. We also need to investigate the storage requirements of our primitives compared to $3 \mathrm{D}$ extensions of realms and dual grids. Thirdly, we have developed a rational number system that allows number representations of arbitrary, finite length and called it RATIO. We have not discussed RATIO in this paper. Thus, in the future, we need to document and present RATIO. Finally, we need to use our primitives to implement the abstract three-dimensional spatial data types in a spatial database system.

\section{REFERENCES}

[1] C. Batut, K. Belabas, D. Bernardi, H. Cohen, and M. Olivier. User's Guide to PARI/GP. The PARI Group, http://pari.math.u-bordeaux.fr/, 2000.

[2] C. Burnikel, R. Fleischer, K. Mehlhorn, and S. Schirra. Efficient Exact Geometric Computation Made Easy. Proc. of the 15th Annual Symp. on Computational Geometry, pp. 341-350, Miami Beach, Florida, USA, June 13-16 1999.

[3] J. A. Cotelo Lema and R. H. Güting. Dual Grid: A New Approach for Robust Spatial Algebra Implementation. GeoInformatica, 6(1):57-76, 2002.

[4] F. Dumortier, M. Gyssens, L. Vandeurzen, and D. Van Gucht. On the Decidability of Semi-Linearity for Semi-Algebraic Sets and Its Implications for Spatial Databases. Proc. of the 16th ACM SIGACT - SIGMOD - SIGART Symp. on Principles of Database Systems, pp. 68-77, Tucson, Arizona, USA, 1997.

[5] F. Geerts and B. Kuijpers. Linear Approximation of Planar Spatial Databases Using Transitive-Closure Logic. Proc. of the 19th ACM SIGMOD - SIGACT - SIGART Symp. on Principles of Database Systems, pp. 126-135, 2000.

[6] R. H. Güting. An Introduction to Spatial Database Systems. VLDB Journal, 3(4):357-399, 1994.

[7] J. Keyser, J. M. Rojas, and K. Ouchi. The Exact Rational Univariate Representation and Its Application. In D. Duffa, R. Janardan, and M. Smid, editors, AMS/DIMACS Volume on Computer Aided Design and Manufacturing. American Mathematical Society/Center for Discrete Mathematics and Computer Science, 2005.

[8] M. Schneider. Spatial Data Types for Database Systems Finite Resolution Geometry for Geographic Information Systems, volume LNCS 1288. Springer-Verlag, Berlin Heidelberg, 1997.

[9] M. Schneider and B. E. Weinrich. An Abstract Model of Three-Dimensional Spatial Data Types. Proc. of the 12th ACM Int. Symp. on Advances in Geographic Information Systems (ACM GIS 2004), pp. 67-72, 2004.

[10] M. Tommila. apfloat: A C++ High Performance Arbitrary Precision Arithmetic Package. World Wide Web, http://www.apfloat.org/apfloat/2.40/, February 22 2003.

[11] C. K. Yap. Towards Exact Geometric Computation. Computer Geometry: Theory and Applications, 7(1-2):3-23, 1997.

[12] S. Zlatanova. On 3D Topological Relationships. Int. Workshop on Database and Expert System Applications, pp. 913-919, 2000. 